\title{
CHEST What's
}

Elizabeth Batalla-Duran

\section{VITAMIN D DEFICIENCY AND EXERCISE CAPACITY IN COPD PATIENTS}

Vitamin D is emerging as a possible factor in airways inflammation in chronic obstructive pulmonary disease (COPD) with deficiency thought to contribute to worsening lung function in COPD patients. A Korean study in Respiration (doi:10.1159/000445266) looked at whether vitamin D deficiency was associated with a more rapid decline in exercise capacity in male COPD patients. Multivariate analysis showed vitamin D deficiency was independently associated with a rapid decline in exercise capacity. The decrease in 6-minute walk distance (6MWD) in patients with severe vitamin $\mathrm{D}$ deficiency was more prominent ( $23.1 \mathrm{~m} /$ year) and the number of patients with moderate-to-severe deficiency was significantly higher in the rapid decliners $(\mathrm{p}=0.002)$. Vitamin D levels were significantly higher in those patients whose exercise capacity improved over the 3 years compared with those with no change or a decline. Studies are needed to ascertain if supplementation with vitamin $\mathrm{D}$ in those with deficiency would benefit the patient in terms of exercise capacity.

\section{SHOULD WE BE LESS PESSIMISTIC ABOUT COPD?}

The Global Initiative for Obstructive Lung Diseases (GOLD) considers COPD to be progressive (with an expected decline in forced expiratory volume $\left(\mathrm{FEV}_{1}\right)$ over time), but a study in PLoS One by de-Torres et al (doi:10.1371/journal.pone.0151856) seeks to challenge this view. The authors studied several years of spirometry data on COPD patients and showed that most patients stay within the same GOLD severity classification or even improve. A total of 535 patients were selected from the BODE international cohort who are followed up at

Correspondence to Dr Elizabeth Batalla-Duran, ST5 Respiratory, Torbay Hospital, Lawes Bridge, Torquay TQ2 7AA, UK; ebatalla-duran@nhs.net least yearly in clinics and are on optimal, standard COPD treatments. They were classed as either 'survivors' (with at least 8 years of spirometry data from follow-up, $\mathrm{n}=318$ ) or 'non-survivors' (with at least 4 years of data before death, $n=217$ ) to reduce survival bias. GOLD grade was measured using $\mathrm{FEV}_{1}$ as per standard GOLD guidelines. Overall, $81 \%$ of the 'survivors' and $79 \%$ of the 'non-survivors' remained in the same GOLD grade during the observation period. Interestingly, $9 \%$ of the survivors and $11 \%$ of the non-survivors showed improvement in GOLD grade. The remainder showed a worsening of their GOLD grade. When patients are diagnosed with COPD, we often paint a picture of a poor prognosis with a deterioration in symptoms over time, but this study indicates we should be a little more positive.

\section{UNFORTUNATELY, NOT A CHEAP TREATMENT FOR IDIOPATHIC PULMONARY FIBROSIS}

Given the expense of the current pharmacological treatments for idiopathic pulmonary fibrosis (IPF) (pirfenidone and nintedanib), a cheap, safe drug, such as a proton pump inhibitor (PPI), would be welcomed by patients and commissioning groups alike. It is speculated that gastrooesophageal reflux is a risk factor for IPF and therefore treatment with antacids could potentially slow progression. However, a study in The Lancet Respiratory Medicine (http://dx.doi.org/ 10.1016/S2213-2600(16)00067-9) failed to show any effect of antacid therapy on progression of IPF. Patients from the placebo groups of three pirfenidone studies were reviewed with post hoc analysis of antacid use. The primary endpoint was disease progression by 1 year, defined as a decrease in predicted FVC by $10 \%$ or more, a decrease in 6 MWD of $50 \mathrm{~m}$ or more, or death. A total of 624 patients were reviewed: 291 were on antacid therapy and 333 were not. At 52 weeks there was no significant difference in disease progression, all-cause mortality or
IPF mortality between the two groups. The incidence of pulmonary infections was, however, higher in those with severe IPF on antacid therapy. The authors conclude that antacid therapy does not improve outcomes in patients with IPF and may actually increase the risk of infections in those with severe disease.

\section{INCREASING INCIDENCE OF NON-TUBERCULOUS MYCOBACTERIA}

There seems to be a never-ending list of non-tuberculous mycobacterium subtypes, not all of which cause clinical symptoms. A British study in BMC Infectious Diseases (doi:10.1186/s12879-016-1521-3) looked at the incidence of non-tuberculous mycobacteria (NTM) in isolates reported to public health authorities between 2007 and 2012. The authors found there has been an almost 10-fold increase in NTM incidence in England, Wales and Northern Ireland since 1995 (interestingly the incidence in Scotland is unchanged). The incidence of pathogenic NTM isolates increased during the period (2007-2012) from 4.8/100 000 to $6.3 / 100000$. In particular, there was an increase in pulmonary NTM, with the commonest NTM being Mycobacterium avium-intracellulare complex (35.6\%), especially in older people ( $>60$ years of age). The authors acknowledge that the data represent patients who have grown the mycobacterium in their samples but may not have clinical manifestations. As to why there has been an increase, they suggest several reasons: an increase in NTMs in the environment, a more elderly population especially with chronic lung disease, the use of inhaled corticosteroids increasing the risk of NTMs, and more clinicians considering it as a possible diagnosis and thus sending samples along with more sensitive testing being available. Whatever the reason, NTMs may be causing an increased burden of disease in the population. It is important to consider NTM in patients presenting with symptoms to allow early identification and treatment.

Competing interests None declared.

Provenance and peer review Commissioned; internally peer reviewed.

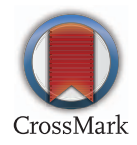

To cite Batalla-Duran E. Thorax 2016;71:674.

Thorax 2016;71:674.

doi:10.1136/thoraxjnl-2016-208905 